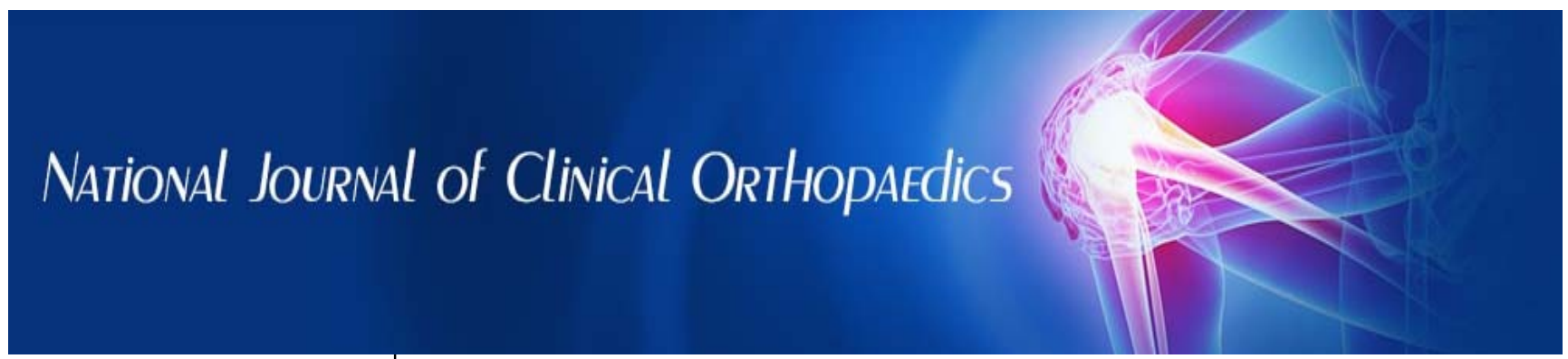

ISSN (P): 2521-3466

ISSN (E): 2521-3474

(C) Clinical Orthopaedics

www.orthoresearchjournal.com

2020; 4(1): 48-52

Received: 15-09-2019

Accepted: 19-11-2019

\section{Dr. Amar}

Senior Resident,

Department of Orthopaedics,

GIMS, Kalaburagi, Karnataka, India

Dr. Syed Azher Hussain

Assistant Professor,

Department of Orthopaedics, GIMS, Kalaburagi, Karnataka, India

\section{Role of posteromedial plating in medial condylar fractures of Tibia}

\section{Dr. Amar and Dr. Syed Azher Hussain}

DOI: $\underline{\text { https://doi.org/10.33545/orthor.2020.v4.i1a.238 }}$

\section{Abstract}

Introduction: The purpose of this study is to define "Role of Posteromedial Plating in medial condylar fractures of Tibia" Therefore, describing the surgical approach to a general orthopaedic surgeon, highlighting the relevant anatomy of posteromedial surgical approach and presenting our experience in treating patients with Posterior tibial shear fracture treating a series of 14 patients using posteromedial approach.

Materials and methods: In our study of 14 patients who sustained high velocity posterior-medial tibial plateau fracture. The mean age was 42 years (range 30 to $50 \mathrm{yr}$ ). Posteromedia surgical approach used for fracture reduction and stabilisation with plate. The patients were followed up at regular interval postoperatively and Oxford Knee Score and Lyshom Score was used to assess final outcome.

Results: 42 (range 40 to 44 ) was the average OKS score at the end of one year. The post-operative clinical measures were early non weight bearing, ROM to reduce post-operative complication \& improve functional outcome. According to oks and lyshom scores the rate of post-operative complications \& functional outcome was significantly better as evident by $100 \%$ showing good to excellent results at the time of full weight bearing.

Conclusion: Buttress/Antiglide plate are usually needed to reduce the fractures anatomically in medial tibial plateau shear fractures which are otherwise irreducible by conventional approaches to achieving absolute stability and mobilise early, NWB, ROM of the knee joint to optimize the functional outcomes and minimise the complications, without the need for revision surgery.

Keywords: Septic arthritis, hip joint, children

\section{Introduction}

Complex tibial plateau fractures are difficult to treat and are associated with posterior shear fractures and pose challenges to a surgeon due to ease of conventional approaches like anteromedial and anterolateral. Due to an inadequate visualization of the fracture zones rather difficult technically to reduce accurately in posterior tibial plateau fractures from the conventional approaches. Thus accurate reduction under visualisation and osteosynthesis with support plate is possible with posteromedial plate through posteromedial approach ${ }^{[1]}$.

The Surgical Treatment of complex tibial plateau fractures with associated posterior shear fractures has been a challenge for an Orthopaedic Surgeon because of his familiarity with only conventional approaches (antero-medial/ antero lateral approaches). Accurate reduction of posterior tibial plateau fractures from the standard approaches is rather difficult technically due to an inadequate visualization of the fracture zone. Thus, posterior approaches to the tibial plateau were introduced through which not only the visualization of the posterior tibial plateau structures but also their osteosynthesis with a support plate is possible.

The purpose of this study is accurate reduction of tibial plateau fracture and maintenance of articular congruity and stable fixation to achieve early ROM, avoid varus/valgus deformity, OA knee, and also repair of all concomitant lesions avoiding menisectomy. ORIF with Plate and screw fixation fulfils all the objectives.

Schatzker type 4 tibial plateau fractures are usually associated with high-energy trauma and these are rare fractures and require high surgical skills to treat as they are highly unstable fractures. Knee joint is subjected to high loads during walking. The total force transmitted across the knee joint in standing position, medial tibial plateau carries $75 \pm 12 \%$. Rigid fixation with posteromedial plating is of almost important in medial condyle fracture because of lack of support to medial side and fibular head that provides lateral support ${ }^{[2]}$.
Corresponding Author: Dr Syed Azher Hussain Assistant Professor, Department of Orthopaedics, GIMS, Kalaburagi, Karnataka, India 
A study has been carried out to evaluate the clinical outcomes and the role of postero medial plating of medial condylar fractures of tibial plateau by ORIF.

\section{Materials and Methods}

This prospective study was conducted in the Department of Orthopaedics, Gulbarga Institute of Medical Sciences from June 2017 to July 2019. A study was performed on 14 adult patients with posteromedial tibial plateau fractures, between the age group of 20-65 years with an average age of 42years. Of these 14patients 12 were male (85.6\%) and 2(14.3\%) were females, 6 out of 14 patients had right sided injury and 8 had left sided injury. Road traffic accident is the mode of injury in all the patients. The information such as sex, age, details of injury, duration and progression were obtained through an interview. All the patients have undergone surgery with ORIF with posteromedial plating. Informed consent will be taken from all the patients before enrolling in the study.

Male and female adult patients with aged more than 18 years having Schatzkers type IV fracture are included in the study. Pathological Fractures of tibial plateau, seriously ill health and polytrauma cases are excluded from study.

Patients were operated using posteromedial buttress plate ( $\mathrm{T}$ or $\mathrm{L}$ plates). All the surgeries were performed in an orthopaedic operation theatre under antibiotic cover. Spinal anaesthesia was administered to them patients. All the surgeries were performed by registered orthopaedic consultants.

\section{Procedure}

Immediate Management: After admission in the emergency department proper history is elicited from the patient. Careful examination performed to rule out other injuries and systemic conditions and all the findings were noted. None of the patients had compartment syndrome or neurovascular injury during admission. Fracture were closed in all patients and the softtissue assessment of injury graded, based on Tscherne-Oestern classification.

The affected limb was temporarily stabilised with use of slab above knee and elevated to reduce oedema. Pain and inflammation were managed using analgesics and antiinflammatory medications. Pre-operative X-ray of and 3D CT were helpful in preoperative planning and to assess the severity of articular depression and cortical split. Routine blood and urine investigation was done. Physician fitness was obtained.

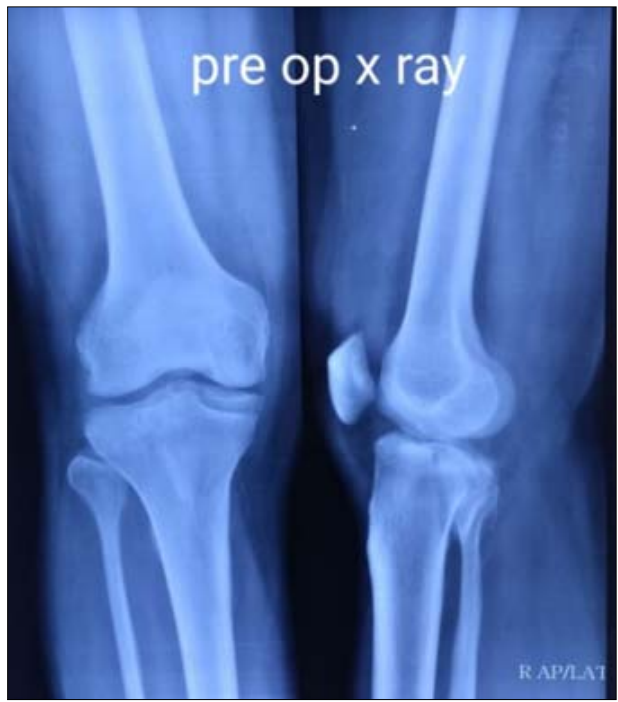

Fig 1: Pre op x-ray

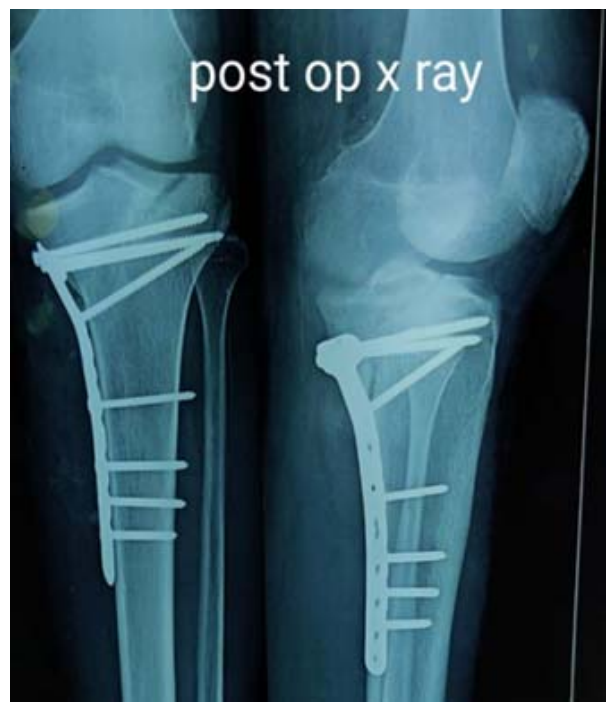

Fig 2: Post op x- ray

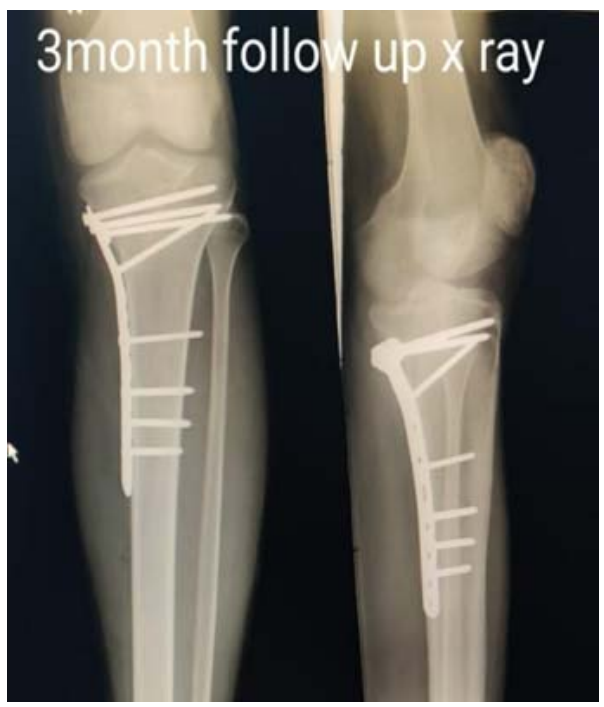

Fig 3: 3 months follow up x-ray

\section{Surgical procedure}

All the surgery was performed between day3 to day12 because time was given settle oedema and assess skin condition. All the surgery were performed under spinal anaesthesia in all cases.

\section{Position and tourniquet}

Patient was put on floating supine position, thigh pneumatic tourniquet is applied after spinal anaesthesia was administered. Contralateral the pelvis rotated towards the injured side hip is flexed, adducted. This helps to put patient in semilateral position and injured leg rotates laterally allows to assess posterior aspect knee and leg. Spinal anaesthesia and sufficient padding and support from the back keep the position easily

\section{Surgical technique}

Postero Medial approach of Wang et al: Incision starts $3 \mathrm{cms}$ proximal to the joint line and follows the postero-medial border of tibia, saphenous nerve and great saphenous vein are identified and retracted along with anterior or posterior flaps.

The Sartorius facia is incised in line with the incision. The Pesanserinus tendons are identified and retracted distal-posterior or proximal -anterior, while medial gastrocnemius and soleus are retracted posteriorly. This exposes the junction between the 
popliteal fascia (posterior and distal), the semi membronus muscle insertion (posterior and proximal), and the MCL. Incise the periosteum sharply, longitudinally down to the bone while staying posterior to the posterior border of MCL. The proximal exposure is limited because of the insertion of the SM. Using sub-periosteal dissection, the insertion of popliteus muscle is elevated off from the posterior tibia, which allows direct visualization of the triangular apex of the fracture at the metadiaphyseal level. During sub-periosteal dissection, there is a chance to avoid injury to the neurovascular structures, including infero medial genicular vessels. Reduction of the joint line is generally done indirectly by direct of the apex and confirmed using fluoroscopic Imaging.

\section{Post-operative care, Follow up}

2 to 16 months follow up done all patients, mean follow up being 9 months. The fracture union took 14 to 32 weeks and average was 25weeks. Range of motion at knee at 12 months follow up was 40 to 120 degrees. Superficial skin infection seen in one patient was settled with appropriate antibiotics and regular dressing. One patient had post traumatic knee stiffness with ROM between 20-100. None of the patients had any other complications like loss of reduction, varus or valgus deformity of knee, DVT, deep infection, OA knee or any neurovascular complications during course of treatment.

\section{Observation and Results}

A study was performed on 14 adult patients with posteromedial tibial plateau fractures, between the age group of 20-65 years with an average age of 42 years (table 2). Out of 14 patients 12 were male and 2 female (table 1). 6 out of 14 patients had right sided injury and 8 had left sided injury (table 3 ). Road traffic accident is the mode of injury in all the patients. 3 patients were immobilised up to 10 days, 8 patients were immobilised up to 3 weeks, 3 patients were immobilised up to 6 weeks (table 4). One patient (7.14\%) showed clinical union during first visit, 9 patient (64.28\%) second follow up at 3months and 4 patient (28.57\%) had union at 6 months. According to oks and lyshom scores the rate of functional outcome was significantly better as evident by over 94\% showing good to excellent results at the time of full weight bearing. The mean OKS score was 42 (range 40to 44) at the final follow up and results been Excellent to Good in $100 \%$ of cases $(14 / 14)$.

Table 1: Sex distribution

\begin{tabular}{|c|c|c|}
\hline Sex & Number of cases & Percentage \\
\hline Male & 12 & $85.7 \%$ \\
\hline Female & 2 & $14.3 \%$ \\
\hline Total & 14 & $100 \%$ \\
\hline
\end{tabular}

In the present study $85.7 \%$ of the patients were males and $14.3 \%$ of the Patients were female.

Table 2: Age distribution

\begin{tabular}{|c|c|c|}
\hline Age & Number of cases & Percentage \\
\hline $20-30$ & 1 & $7.1 \%$ \\
\hline $30-40$ & 8 & $57.1 \%$ \\
\hline $40-50$ & 4 & $28.57 \%$ \\
\hline $50-65$ & 1 & $7.14 \%$ \\
\hline Total & 14 & $100 \%$ \\
\hline
\end{tabular}

Mean age of the study 42 years
Table 3: Laterality

\begin{tabular}{|c|c|c|}
\hline Side & Number of cases & Percentage \\
\hline Right & 6 & $42.85 \%$ \\
\hline Left & 8 & $57.14 \%$ \\
\hline Total & 14 & $100 \%$ \\
\hline
\end{tabular}

Table 4: Period of immobilisation

\begin{tabular}{|c|c|c|}
\hline Period of immobilisation & Number of cases & Percentage \\
\hline Up to 10 days & 3 & $21.42 \%$ \\
\hline Up to 3 weeks & 8 & $57.14 \%$ \\
\hline Up to 6 weeks & 3 & $21.42 \%$ \\
\hline Total & 14 & $100 \%$ \\
\hline
\end{tabular}

Table 5: Clinical union

\begin{tabular}{|c|c|c|}
\hline Follow up & Number of cases & Percentage \\
\hline First (6 weeks) & 1 & $7.14 \%$ \\
\hline Second (3 months) & 9 & $64.28 \%$ \\
\hline Third (6 months) & 4 & $28.57 \%$ \\
\hline Total & 14 & $100 \%$ \\
\hline
\end{tabular}

Table 6: Oxford knee score

\begin{tabular}{|c|c|c|}
\hline Score & Number of cases & Percentage \\
\hline Score $0-19$ & 0 & $0 \%$ \\
\hline Score $20-29$ & 0 & $0 \%$ \\
\hline Score $30-39$ & 0 & $0 \%$ \\
\hline Score $40-48$ & 14 & $100 \%$ \\
\hline Total & 14 & $100 \%$ \\
\hline
\end{tabular}

Table 7: Lysholm score

\begin{tabular}{|c|c|c|}
\hline Score & Number of cases & Percentage \\
\hline Excellent & 12 & $85.71 \%$ \\
\hline Good & 2 & $14.28 \%$ \\
\hline Fair & 0 & $0 \%$ \\
\hline Poor & 0 & $0 \%$ \\
\hline Total & 14 & $100 \%$ \\
\hline
\end{tabular}

Table 8: Complications

\begin{tabular}{|c|c|c|}
\hline Complications & Number of cases & Percentage \\
\hline Knee stiffness & 1 & $7.14 \%$ \\
\hline Wound discharge & 1 & $7.14 \%$ \\
\hline No complications & 12 & $85.71 \%$ \\
\hline Total & 14 & $100 \%$ \\
\hline
\end{tabular}

\section{Discussion}

The tibial plateau fractures are quite rare and unstable require accurate reduction to maintain articular congruity and stable fixation to start early rom and provide stability. And also avoids varus and valgus deformity. Open operative technique also helps in repair of concomitant lesions and avoids development of osteoarthritis of knee joint knee. All these objects are best fulfilled by ORIF with Plate and screw fixation. Schatzker type 4 tibial plateau fractures are usually associated with high-energy trauma and these are rare fractures and require high surgical skills to treat as they are highly unstable fractures. Knee joint is subjected to high loads during walking. The total force transmitted across the knee joint in standing position, medial tibial plateau carries $75 \pm 12 \%$. Rigid fixation with posteromedial plating is of at most important in medial condyle fracture because of lack of support to medial side and fibular head that provides lateral support ${ }^{[2]}$.

The classifications of J. Schatzker (1978) ${ }^{[2,15]}$ are the most used ones for treatment of tibial plateau fractures, but neither considers what parts of the tibial condyles are injured. Therefore 
this classification cannot be used for posterior tibial plateau fractures. There are many surgical approaches to address the complex posterior tibialplatue fractures associated with posterior shear fractures. A midline approach with division of the patellar ligamentor even an osteotomy of the tibial tuberosity are used to treat these fractures but are associated with complications related to wound healing and seems very hostile for these fractures ${ }^{[3,4,11]}$.

Posteromedial approach first described by lobenhoffer and later Fakler and Brunner developed direct posterior approach to address this main fragment ${ }^{[5-9]}$. The posteromedial, posterior or the avulsed tibialeminence, which we observed in all cases, but difficult to reduce and fix. And very difficult to address posterolateral depression. Georgiadis recommended combined anterior and posterior approaches to align all fracture fragments [10]. Extensive soft-tissue dissection, several incisions and limited access to all fracture elements are the major disadvantages of these approaches. According to study conducted by Barei et al., ${ }^{[12]} 30 \%$ of fractiures are posteromedial in bicondylartibialplatue fractures. Higgins et Higgins et al., ${ }^{[13]}$ In his study of 111 bicondylartibial plateau fractures, $59 \%$ of fractures that is 65 cases were having posteromedial tibial plateau fractures on CT scan. Avulsion of semimembranosus tendon insertion site due to the stress on the knee joint in hyper extension results in posteromedial tibial plateau fragment and fragment is relatively big according to some authors ${ }^{[13]}$. A study by Barei et al., ${ }^{[12]}$ shows that fracture fragment constitutes $58 \%$ of medial plateau surface and 23\% (8$47 \%$ ) of the entire plateau articular surface. According study by Higgins et al., ${ }^{[13]} 25 \%$ is the average area of bone fragment of entire tibial platuea articular surface

Hsieh et al., ${ }^{[14]}$ placed lag screws from anterior to posterior for fixation of posteromedial tibial plateau fracture in 8 cases and the results were satisfactory. But most authors believed that it was prone to loss of reduction with the only use of lags crews when the fracture fragment was big.

Yoo et al., ${ }^{[20]}$ revealed that posteromedial tibial plateau fracture fragments tolerated higher loads by using the lateral $3.5-\mathrm{mm}$ conventional non-locking proximal tibial plate and posteromedial 1/3 tubular plate fixation. Posteromedial plateau fractures, medial fracture dislocations and wedge-like posteriormetaphy seal fractures are best managed from a posteromedial approach. The straight posterior approach is rarely indicated, but works well for isolated posterior shear fractures, PCL avulsion and posterior fracture dislocations. The study of these injuries demonstrates that the use of anterior approaches that are most frequently used in such clinical situations was not sufficiently efficient due to an inadequate

Visualization of the fracture zone, extreme difficulty of performing anatomic reduction of the articular tibial surface and impossibility of support plate implantation. A number of posterior approaches have been described in the special literature that allow for visualization and adequate fixation of all the tibial plateau parts. The variants of the posterior and posteromedial approaches are used for osteosynthesis of the posterior medial tibial condyle parts. However, the analysis of the available publications shows that these approaches are technically difficult. They do not eliminate the risk of damage to large vessels and nerves as well as they are insufficiently substantiated from topographic and anatomic positions. Therefore, the substantiation and improvement of the technique of performing posterior approaches to the knee in the patients of the mentioned profile as well as specifying the indications for each of them appear, to be promising directions of research in the area under consideration. The limitation of this study is that the sample size is quite small. Although it was found that postoperative complications were low and short-term prognosis was satisfactory, we cautiously concluded that posteromedial approach conceived of significant value for the management of medial tibial plateau fractures. On the prerequisite of excellent reduction and fixation, weincline to adopt a single posterior approach to deal with tibial plateau fractures. The results in our series of 14 patients at a mean follow up of 24 months (range 5 to 30 ) all 14 were having (100\%) excellent, results (graded as per OKS). Our results are superior / comparable to most of the studies of reported in the literature as shown in the table above. All the patients (14 of 14) were satisfied at mean follow up of 24 months, although a little prolonged rehabilitation period required in most of our patients following their surgery. However none of our patients experienced any instability of the knee or a poor functional result.

The results this study demonstrate that posteromedial plating to stablise the posterior shear fractures of tibial plateau produce very satisfactory out-comes.

\section{Conclusion}

A posterior/ postero medial approach for posterior tibial plateau shear fractures (which are otherwise irreducible by conventional approaches) and buttress/antiglide plate are usually needed to reduce the fractures anatomically, achieving absolute stability and mobilise early NWB, ROM of the knee joint to optimize the functional outcomes and minimise the complications, without the need for revision surgery.

\section{References}

1. Belen'kii IG, Kochish A, Iu Kislitsyn MA. Fractures of the tibial condyles: current treatment methods and surgical approaches (literature review). Genij Ortopedii 2016;(4):114-122.

2. Schatzker J, McBroom R, Bruce D. The tibial plateau fracture: the Toronto experience 1968-1975. Clin Orthop 1979;138:94-104.

3. Espinoza-Ervin CZ, Starr AJ, Reinert CM et al. Use of a midline anterior incision for isolated medial tibial plateau fractures. J Orthop Trauma 2009;23:148-53.

4. Fernandez DL. Anterior approach to the knee with osteotomy of the tibial Tubercle for bicondylartibial fractures. J Bone Joint Surg Am 1988;70:208-19.

5. Brunner A, Honigmann P, Horisberger M, Babst R. Open reduction and fixation of medial Moore type II fractures of the tibial plateau by a direct Dorsal approach. Arch Orthop Trauma Surg 2009;129:1233-8.

6. Fakler JK, Ryzewicz M, Hartshorn C et al. optimizing the management of MooreType I postero-medial split fracture dislocations of the tibial head: description of The Lobenhoffer approach. J Orthop Trauma 2007;21:330-6.

7. Galla M, Lobenhoffer P. The direct, dorsal approach to the treatment of unstable tibial posteromedial fracturedislocations. Unfallchirurg 2003;106:241-793.

8. Lobenhoffer $\mathrm{P}$, Gerich $\mathrm{T}$, Bertram $\mathrm{T}$ et al. Particular posteromedial and posterolateral approaches for the treatment of tibial head fractures. Unfallchirurg 1997;100(12):957-67.

9. Tscherne H, Lobenhoffer P. Tibial plateau fractures. Management and expected results. Clin Orthop Relat Res 1993;292:87-100.

10. Georgiadis GM. Combined anterior and posterior approaches for complex tibial plateau fractures. J Bone Joint Surg Br 1994;76:285-9.

11. Gösling, Krettek C et al. Less invasive stabilization of 
complex tibial plateau fractures: a biomechanical evaluation of a unilateral locked screw plate and double plating. J Orthop Trauma 2004;18:546-51.

12. Barei D, O’Mara T, Taitsman L, Dunbar RP, Nork SE. Frequency and fracture morphology of the postero-medial fragment in bi-condylar tibial plateau fracture patterns. J Orthop Trauma 2008;22:176-82.

13. Higgins TF, Kemper D, Klatt J. Incidence and morphology of the posteromedial fragment in bi-condylar tibial plateau fractures. J Orthop Trauma 2009;23:45-51.

14. Hsieh CH, Huang HT, Liu PC, Lu CC, Chen JC, Lin GT. Anterior approach for posteromedialtibial plateau fractures. Kaohsiung J Med Sci 2010;26:130-5.

15. Bendayan J, Noblin JD, Freeland AE. Posteromedial second incision to reduce and stabilize a displaced posterior fragment that can occur in schatzker $\mathrm{V}$ bicondylar tibial plateau fractures. Orthopedics 1996;19:903-4.

16. Bhattacharyya T, McCarty 3rd LP, Harris MB, Morrison SM, Wixted JJ, Vrahas MS et al. The posterior shearing tibial plateau fracture. Treatment and results via a posterior approach. J Orthop Trauma 2005;19:305-10.

17. Furey A, Floyd JC, O’Toole RV. Treatment of tibial plateau fractures. Curr Opin Orthop 2007;18:49-53.

18. Delamarter R, Hohl M: The cast brace and tibial plateau fractures, Clin Orthop 1987;242:26.

19. Hong-wei Chen, Jun Pan et al. A posteromedial approach for open reduction and internal fixation of posteromedial tibial plateau fracture. Acta Orthop Belg 2016, P258-264.

20. Yoo BJ et al. Stabilization of the posteromedial fragment in bicondylartibial plateau fractures: a mechanical comparison of locking and non-locking single and dual plating methods. J Trauma 2010;69(1):148-55. 